\title{
1 Fungal-bacterial interaction selects for quorum sensing mutants and a metabolic shift 2 towards the production of natural antifungal compounds
}

4 Andrea G. Albarracín Orio ${ }^{1,2,3}$, Daniel Petras 3 , Romina A. Tobares ${ }^{2}$, Alexander Aksenov ${ }^{3}$, Mingxun Wang 3 , 5 Florencia Juncosa ${ }^{1}$, Pamela Sayago ${ }^{1}$, Alejandro Moyano², Pieter C. Dorrestein ${ }^{3}$, Andrea M. Smania ${ }^{2}$ and 6 Daniel A. Ducasse ${ }^{1,4}$

7

8 'IRNASUS, Universidad Católica de Córdoba, CONICET, Facultad de Ciencias Agropecuarias. Av. Armada 9 Argentina 3555 (X5016DHK) Córdoba, Argentina. ${ }^{2}$ Universidad Nacional de Córdoba. Facultad de Ciencias 10 Químicas. Departamento de Química Biológica Ranwel Caputto. Córdoba, Argentina. CONICET. 11 Universidad Nacional de Córdoba. Centro de Investigaciones en Química Biológica de Córdoba 12 (CIQUIBIC), Córdoba, Argentina. ${ }^{3}$ Collaborative Mass Spectrometry Innovation Center, 9500 Gilman Drive, 13 University of California, San Diego, CA 92093, USA. ${ }^{4}$ Instituto de Patología Vegetal IPAVE-CIAP, Instituto 14 Nacional de Tecnología Agropecuaria, Camino 60 Cuadras Km $5^{1 / 2}$ (5019) Córdoba, Argentina.

15 To whom correspondence may be addressed. Email: asmania@fcq.unc.edu.ar, 16 a.albarracinorio@conicet.gov.ar.

\section{Abstract}

Metabolic exchange may define adaptation to the changes imposed by microbial interactions. We found that antifungal activity of cell-free supernatants of Bacillus subtilis ALBA01 is acquired after interacting with the soil fungus Setophoma terrestris (ST). Here we evaluated different traits of $B$. subtilis ALBA01 before (pre-ST) and after (post-ST) interacting with the fungus in co-cultures and inquired about the molecular mechanisms underlying this inter-kingdom interaction dependent antagonistic activity. Apart from the high fungal inhibition activity, we observed that the ability to form robust biofilms in vitro was a prevailing feature of post-ST and that biofilm formation was positively correlated to biocontrol efficacy. Moreover, nuclear magnetic resonance and mass spectrometry analysis revealed a differential metabolomics profile in postST variants, including the unexpected absence of surfactin or plipastatin production. Disruption of the srfAA gene implicated in surfactin synthesis resulted in an abolition of the ST-driven antifungal activity and ST growth inhibition levels similar to those observed for post-ST variants. The post-ST phenotype was stable after several passages on solid media, indicating that a mutation-based process underlies this phenotypic adaptation process. In fact, whole genome comparison revealed mutations that converge in genes of the 
ComQPXA quorum sensing system in post-ST variants. Altogether, our results suggest a role for a still unknown quorum sensing regulated pathway, which by classical antimicrobial lipopeptide abrogation mediates $B$. subtilis ALBA01 adaptive phenotypic variation and that may be responsible for the antagonistic behavior it exerts on S. terrestris.

\section{Introduction}

The immense microbial diversity within the soil leads to extensive interspecies interactions (1). Generation of antagonistic and mutualistic behaviors, mediated by exchange of small diffusible secondary metabolites, facilitates microbial adaptation to complex communal life. Microorganism co-cultures are inspired by the natural microbe communities that are omnipresent in nature. It has been shown that microorganism coculturing can stimulate silent gene clusters, but the molecular mechanisms that can prompt this phenomenon remain unknown. It has been reported that co-culturing Aspergillus nidulans with soil-dwelling actinobacteria result in the expression and production of specialized fungal metabolites (2). Moreover, co-culturing of Streptomyces lividans TK23 with Tsukamurella pulmonis TP-B0596 induce the production of a still unidentified red pigment, while $S$. endus S-522 co-cultured with T. pulmonis TP-B0596 produces the novel antibiotic alchivemycin A65 (3). The compounds secreted by microbes in response to species interactions can frequently antagonize other microorganisms, have roles as intraspecific signaling cues, alter general microbial development and/or their confinement in particular environmental niches $(4,5)$.

Bacillus subtilis, responds to distinct signals by differentiating into specialized cell types (6), which are related to its survival in the environment. Indeed, by responding to quorum sensing molecules, B. subtilis can undergo several developmental processes such as formation of biofilms, within which a subpopulation of cells differentiates to produce an extracellular matrix that encases the community

$54(7,8)$. As a relevant molecule in B. subtilis, surfactin is a lipopetide biosurfactant and antibiotic that displays hemolytic, antiviral, antimycoplasma and antibacterial activities, for example against the plant pathogen Pseudomonas syringae $(9,10)$. It has been reported that production of surfactin is essential for biofilm formation and general medium/niche colonization capacity in $B$. subtilis (11-13). The production of surfactin

58 is quorum-sensing dependent and is activated by the end of the exponential growth phase (14). 59 Transcription of srfA operon, which encodes surfactin production genes, is directly activated by the 
phosphorylated form of the master regulator ComA, which is part of the ComQXPA quorum-sensing system (14-16). In this system, the ComX autoinducer, encoded by the comX gene and post-translationally cleaved and prenylated by ComQ, binds to and activates ComP, a membrane-anchored histidine kinase receptor, which then phosphorylates the response regulator ComA that finally activates transcription of more than one hundred genes $(17,18)$. Modifications in the regulation of srfA operon directly affect the expression of the competence regulatory gene coms, which resides within the same operon, and thus alter competence, sporulation and motility regulation pathways (19). In fact, ComS causes the release of the factor ComK that also activates the transcription of several genes involved in DNA cleavage, transformability, motility, arginine biosynthesis and transport (20). On the other hand, it has also been reported that ComA activates the expression of $\operatorname{deg} Q$ gene in undomesticated $B$. subtilis strains, an essential gene for the synthesis of another non-ribosomal peptide, plipastatin $(21,22)$. Plipastatin, a member of the fengycin family of lipopetides, is a powerful antifungal compound with a specific and strong activity against filamentous fungi (23-26). All these evidences highlight the complex and refined surfactin-mediated connection among development pathways in $B$. subtilis. It has been reported that cellular differentiation and biofilm formation in $B$. subtilis can be affected not only by intraspecific but also by interspecific signals and that metabolites secreted by other soil microbes different from Bacillus spp. might alter biofilm development in $B$. subtilis (27). For example, nystatin, amphotericin, valinomycin and gramicidin can all induce the quorum-sensing pathway and cause differentiation of the subpopulation of matrix producers (28). The ability to sense small molecules produced by a range of diverse soil microorganisms suggests a broad mechanism that $B$. subtilis uses to respond to neighboring communities by altering its development. Thus, it is important to complement our current knowledge on the interspecies communication strategies of rhizosphere B. subtilis because systems involved in development programs can regulate the expression of antipathogenic and biocontrol agents like surfactins and biofilm-inducing compounds (29).

We have previously shown that Bacillus subtilis ALBA01, a strain isolated from the rhizosphere of onion 84 plants, is capable of strongly inhibit the growth of S. terrestris in vitro (30), one of the most severe pathogens of onion and the causative agent of its major limiting diseases, the pink root. Interestingly, we found that BsA01 acquires a strong antagonistic behavior against ST after previous interaction with the fungus in co- 
factors since it is observed in cell-free supernatants of BsA01 previously grown in the presence of the fungus (post-ST variants) but not in those supernatants obtained from variants grown without any previous contact with it (pre-ST variants) (30). It is noteworthy that this behavior appeared to be specifically provoked by the interaction with $S$. terrestris since no antagonistic activity against two other onion pathogens, Fusarium oxysporum and F. proliferatum, was observed (30). In order to gain insights into the underlying mechanisms responsible for this behavior, we analyzed differentiation processes of the bacterium that lead to the release of diffusible bacterial inhibitory compounds. In this study, we combined phenotypic characterization, untargeted LC-MS/MS and NMR-based metabolomics approaches and comparative genomics to distinguish the differences between the pre and post-ST variants. Our findings suggest that a mutation-based phenotype variation involving quorum sensing genes arises in BsA01 after co-culturing with S. terrestris and mediates both a suppression of surfactin and plipastatin production and a metabolomics profile change that might be the bases of the specific antagonistic behavior and the stability of the phenomenon.

\section{Results}

102 Bacillus subtilis post-ST variants undergo phenotypic variation and form robust biofilms

103 By co-culturing assays, we found that after 15 days of interaction with the soil fungal pathogen S. terrestris

104 (ST), the strain of $B$. subtilis ALBA01 acquires a strong antagonistic activity mediated by secreted factors, since fungal growth inhibition is observed in bacterial cell-free supernatants (30) (Figure $1 \mathrm{~A}$ and $\mathrm{B}$ ). These variants were named as post-ST to distinguish them from those variants grown without previous contact

107 with the fungus (pre-ST variants) and whose cell-free supernatants do not display antagonistic activity (30)

108 (Figure 1B). To gain further insight into the S. terrestris-driven bacillus behavior, we first performed a 109 phenotypic characterization of both, the pre-ST and the post-ST variants. As shown in Figure 1C, the post-

110 ST variants exhibited changes in morphology with an apparent increase in roughness and wrinkly colony

111 phenotype relative to $B$. subtilis colonies grown without previous contact with the fungus (Figure 1C, panel

1124 and 5). A qualitative analysis by electron microscopy of $B$. subtilis after co-culture with $S$. terrestris 113 revealed a high number of longer cells as well as a reduced number of sporulated cells in comparison to 114 control pre-ST cells (Figure 1C, panel 6). We then characterized the capacity to form biofilm in both, pre- 
115 ST and post-ST variants. In contrast to general assumption that soil microorganisms might release compounds that would inhibit rather than stimulate cellular differentiation in $B$. subtilis, we observed that

117 both pre and post-ST variants were capable of forming floating biofilms (pellicles) characteristically

118 associated with biofilm formation in LBGM medium (31). However, the pre-ST variants formed a more 119 featureless biofilm with slightly thinner pellicles that easily disintegrate (Figure 2A). In contrast, the post-ST 120 variants were able to develop into more wrinkled and thicker pellicles, which is consistent with a robust 121 biofilm formation (Figure $2 \mathrm{~A}$ ). We also evaluated the ability of the $B$. subtilis variants to form submerged 122 biofilms on borosilicate surfaces and quantified biofilm formation by crystal violet staining. As shown in 123 Figure 2B, biofilm formation was significantly increased in post-ST variants. Moreover, post-ST lacked 124 swarming motility when spotted in semisolid LB medium, while pre-ST colonized the entire Petri dish 125 showing full swarming motility (Figure 2C). Remarkably, when re-inoculated onto a fresh growth medium 126 (passaging) the post-ST variants still maintained not only the structured colony morphology but also the 127 increased capacity of biofilm formation, indicating that $B$. subtilis experiences a genetic stable phenotypic variation after co-culture with $S$. terrestris. metabolite profile in post-ST variants

131 To gain deeper understanding on the differential antifungal activity observed in the cell-free supernatants 132 of the post-ST variants, we applied an untargeted metabolomics profiling based on Proton Nuclear Magnetic 133 Resonance ( $\left.{ }^{1} \mathrm{H}-\mathrm{NMR}\right)$ and multivariate data analysis tools to characterize the difference in secondary 134 metabolite profile of cell-free supernatants of the BsA01 pre-ST and post-ST variants. Twelve samples of cell-free bacterial supernatants were analyzed, six of which belonged to the pre-ST variant group and the 136 other six to the post-ST variant group. Principal Component Analysis (PCA) was applied to the data 137 obtained from NOESY spectra of each sample. As shown in Figure 3A, samples from the pre-ST group 138 clustered together and clearly separated from post-ST variants samples. To discard potential biases in sample separation as a consequence of the sample run order, the samples were analyzed by varying the

140 initial order and we found the same grouping profile as observed before (Figure S1a). Moreover, by 141 Orthogonal partial least squares discriminative analysis (OPLS-DA) we could maximize the separation 
142 between both groups (Figure S1b). To visualize the differences between classes, we generated S-line plots

143 in OPLS-DA models and observed different signals that represent discriminant metabolites between both

144 BsA01 pre-ST and post-ST groups of samples (Figure 3B), all or some of which might constitute the factors

145 responsible for the antagonistic antifungal behavior.

146 We then compared the chemotypes of whole cells of BsA01 pre and post-ST variants to test whether the 147 metabolome is significantly altered between both variants. We carried out a non-targeted liquid chromatography tandem mass spectrometry (LC-MS/MS) based metabolomics analysis and observed a clear separation in the global metabolome between the variants in a Principal Coordinate Analysis (PCoA) (Figure 4A). According to random forest analysis, lipopetides surfactin and plipastatin appeared to be the most important variables that determine the difference between pre- and post-ST variants (Figure 4B).

152 Intriguingly, the post-ST variants showed a marked reduction in the intensity of surfactin ions $(\mathrm{m} / \mathrm{z} 1008.66$; $1531022.67 ; 1036.69 ; 1044.65 ; 1058.67)$ and also a decrease in the intensity of peaks with masses indicative 154 of the plipastatin (Figure 4C and D, respectively), both of which were identified through spectrum library 155 matching and spectral networking $(32,33)$. Moreover, the post-ST variants clustered together with a $B$. 156 subtilis NCBI 3610 surfactin-defective mutant, highlighting the role of surfactin in the separation between 157 the groups (Figure 4A). Figure S2 shows the molecular network obtained by analysis of the chemical profile 158 of the BsA01 variants. The pie charts in the network indicate relative abundances of ion features between 159 the different groups. The molecular networks indicate that not only the known surfactin and plipastatin derivatives were diminished in post-ST variants but also other related unknown derivatives. In a second approach, we applied LC-MS/MS based metabolomics to analyze cell-free supernatants of the BsA01 pre 162 and post-ST variants. In accordance with the results found for the whole bacterial cell analysis, we observed 163 a clear discrimination according to the origin of the cell-free supernatants with PcoA plots showing that post-ST samples clustered separated from pre-ST variants (Figure 5A). Moreover, for post-ST cell-free 165 supernatants we could not detect surfactin (Figure 5B) nor plipastatin (Figure 5C) ions in levels comparable 166 to those found in the pre-ST variant of $B$. subtilis ALBA01 strain. Consistent with this, we also found that 167 post-ST variants did not show hemolytic activity (Figure 5D). Taking together, these results indicate that 168 what separates the post-ST variants most is the absence/decrease of the antimicrobial compounds 169 surfactin and plipastatin. In this sense, the abrogation of surfactin production in the post-ST variants is even 
most interesting since this lipopeptide can be considered a key player in environmental adaptation processes, quorum-sensing and biofilm formation in B. subtilis.

\section{Biofilm is positively correlated with the antifungal activity of cell-free supernatants}

173 So far, our findings suggest that the biofilm formation and the absence of the lipopetide surfactin have a 174 role in the antagonistic behavior of the post-ST variants. Biofilm formation depends on the synthesis of

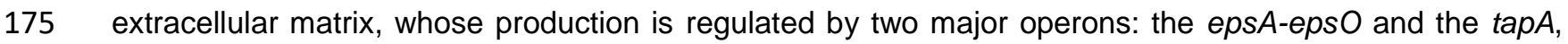
176 both under the control of the transcriptional repressor and biofilm master regulator $\operatorname{SinR}(34,35)$. On the 177 other hand, surfactin, which is involved in swarming motility and also acts as an antimicrobial metabolite $(9$, 178 36), has been identified as a communication signal that triggers biofilm formation $(6,28)$. To test if key 179 genes involved in biofilm formation and surfactin production are related to the antifungal activity acquired 180 by post-ST variants, we generated two BsA01 mutants: a hyper robust biofilm-forming $\Delta \sin R$ and a 181 surfactin-defective producer $\triangle$ srfAA. The activity of the BsA01 mutants against $S$. terrestris was screened 182 by 15-day co-cultures on PDA dishes before and after which samples of each mutant were recovered and 183 tested. When cell-free supernatants of $\Delta \sin R$ pre-ST mutant were examined for antifungal activity, although 184 lower than BsA01 pre-ST variant, it displayed a significantly higher activity than cell-free supernatants 185 obtained from $\Delta \sin R$ post-ST cells (Figure 6). While this result suggest that there is a positive correlation 186 between biofilm formation and antagonism, the mere activation of the biofilm pathway cannot completely 187 explain the ST-driven behavior of BsA01. On the contrary, antifungal activity differences between pre-ST 188 and post-ST supernatants disappeared when the $\triangle$ srfAA surfactin-less mutant was analysed (Figure 6). 189 Moreover, the absence of surfactin per se led to the acquisition of an equalled mycelium growth inhibitory 190 activity with respect to the BsA01 post-ST variants that previously interacted with the fungus (Figure 6). 191 The inability to produce and release surfactin not only resulted in an enhanced level of antagonism towards 192 ST but also in a total suppression of the ST-driven phenomenon.

\section{3 \\ B. subtilis post-ST variant undergoes a mutation-based phenotypic variation}

194 To gain insight into the mechanisms underlying the specific and stable phenotypic variation in $B$. subtilis 195 ALBA01 directed by the interaction with S. terrestris, we decided to sequence the whole genome of three 
different post-ST variants (post-ST1, post-ST2 and post-ST3), which were independently derived from the ALBA01 strain that was taken as a pre-ST ancestor and used as a reference. Reads from each post-ST variant were aligned against the ALBA01 genome (NCBI Bioproject PRJNA316980) to assess the genetic changes accumulated in post-ST during the interaction with the fungus. Overall the complete genomes, we found between one and three different mutations in coding sequence regions for post-ST variants (Table 1). Surprisingly, each one of them carried at least one mutation in genes of the ComQXPA quorum-sensing system. Among these, we found that post-ST 1 and post-ST 2 variants hold a mutation in the coding sequence of the comA gene, whereas the remaining post-ST 3 variant carried a mutation in the kinase encoding gene, comP. For both post-ST 1 and 2 variants, mutations in comA resulted in an insertion of one A in a homopolymeric tract of six adenines at position 415 of the gene $\left(+1 A_{415}\right)$, causing a frameshift mutation and the generation of a stop codon in position 157 of the protein sequence. Structurally, ComA belongs to the NarL family of transcriptional activators with a C-terminal DNA-binding domain that shares sequence homology with the quorum sensing regulator LuxR of Vibrio fischeri, and is a member of the widespread tetrahelical helix-turn-helix $(\mathrm{HTH})$ subfamily (17). The $\mathrm{N}$-terminal domain, which comprises the response regulator receiver motif (sites 3 to 121), includes the phosphorylation site D55, and the loop linker sites with the residue T141. The C-terminal region contains the Helix-Turn-Helix motif (sites 147 to 212) and within this the DNA binding domain (171 to 190). According to prediction analysis by InterPro (37), the $+1 \mathrm{~A}_{415}$ mutation may lead to a truncated version of the protein that lost the expected DNA-binding domain. On the other hand, the mutation in the comP gene in post-ST3 variant consisted of a 100 bp-deletion at position 632 of the gene and is predicted to cause the loss of the complete histidine kinase domain of the protein. It is important to mention that the presence of these mutations was confirmed by PCR amplification of comA and comP followed by direct sequencing. We then aimed to explore the involvement of com mutations in the post-ST phenotype and to inquire to what extent com genes could be mutated in BsA01 after interacting with $S$. terrestris. For that, we conducted a whole-genome sequencing of a pool of $15 B$. subtilis post-ST variants independently obtained after interaction with the fungus in co-culture (Pool-seq). To identify allelic variations in the pool, we first mapped the sequence reads to the reference genome ( $B$. subtilis ALBA01, NCBI Bioproject PRJNA316980) and then performed the variant calling (see Material and Methods). Surprisingly, we identified six different mutations in the comQXPA operon within the population 
224 of post-ST variants, revealing a marked convergence of mutation within these genes that might explain the

225 metabolomics changes observed. Remarkably, we found the previously described comA insertional

226 mutation and also three new mutations in comA, two substitutions, T215A and C601T, and one 5

227 nucleotide-insertion at position 299 (Table 2). The T215A mutation generated a premature stop codon at

228 position 126 of ComA whereas the C601T and the 5 nucleotide-insertion generated stop codons at position

229201 and 78 of the protein, respectively. Moreover, two new mutations in the comP gene were observed,

230 and both of them represented insertions. The GC insertion at position 1517 of the gene generated a

231 truncated version of ComP with a stop codon at postion 512, and the insertion of AT produced a stop codon

232 at position 232 of ComP (Table 2). These findings present evidence of a mutation-based phenotypic change

233 in the post-ST variants and support the hypothesis that the environmental conditions that B. subtilis ALBA01

234 encountered when interacting with $S$. terrestris may force an adaptive process that allows the bacterium to

235 resist and outperform the fungus and that involved a not yet fully understood pathway controlled by

236 ComQXPA.

\section{Discussion}

238 The soil-dwelling bacterium Bacillus subtilis and closely related species have been extensively used as 239 biological control agents in agriculture, mainly due to their production of antibiotics with a great variety of 240 chemical structures (10). In contrast, relatively little is known about the molecular mechanisms underlying

241 the $B$. subtilis biocontrol behavior. A typical mechanism of competition among microorganism is the 242 secretion of metabolites that produce diverse effects during intra-, inter-species and even inter-kingdom 243 interactions. In this sense, B. subtilis, secretes numerous secondary metabolites including the antibiotic 244 compound subtilin, the bacteriocin subtilosin, lipopeptides as surfactins, plipastatins, and others, and the 245 polyketide/nonribosomal peptide hybrid compound bacillaene (25). Many of these metabolites play dual 246 roles as antimicrobial compounds and signaling molecules during interactions, participating in development 247 modulation, biofilm formation and inhibition of virulence factors of competitor individuals $(4,26,38)$. In a 248 previous work, we isolated a strain of $B$. subtilis subsp. subtilis from the rhizosphere of onion plants, BsA01, 249 that is capable of strongly inhibiting the growth of the soil-borne fungal pathogen S. terrestris in vitro (30). 250 Remarkably, the antagonistic behavior of BsA01 is manifested in cell-free supernatants of cells previously 
251 grown in the presence of the fungus (post-ST variants) but not in cells never exposed to the fungus (pre-

252 ST variants), indicating that the interaction with S. terrestris promotes a bacterial transformation that leads

253 to the secretion of antifungal factors. In this work, we aimed to understand this phenomenon through an

254 exhaustive characterization of BsA01 post-ST variants that included phenotypic, genetic, genomics and metabolomics analysis. We present evidences that after 15 days of co-culture with $S$. terrestris, BsA01 exhibits changes in colony morphology and acquires the ability to form robust biofilms. In fact, $B$. subtilis post-ST variants display a remarkable increase in colony roughness/wrinkles and highly structured biofilm pellicles in the air-liquid medium interphase. We also observed that post-ST variants show neither hemolytic activity nor swarming motility indicating an absence of surfactin or the impossibility of producing this lipopeptide. In agreement with these findings, we observed that both a BsA01 hyper biofilm-forming $\Delta \sin R$ mutant and a surfactin-deficient $\triangle$ srfAA mutant display higher antifungal activity when compared to the pre-

262 ST variant. Is noteworthy that the sole absence of surfactin in the $\triangle$ srfAA mutant was capable of erasing 263 the differences in activity that pre and post-ST cell-free supernatants exhibited toward S. terrestris. 264 Furthermore, LC-MS/MS-based metabolomics expose major differences in the metabolomes of pre and post-ST variants, with post-ST showing a marked reduction or absence of surfactin ions. Interestingly, PcoA analysis showed that post-ST variants clustered both together and with a $B$. subtilis NCBI 3610 knock-out surfactin derivative mutant suggesting that key differences between the BsA01 variants might rely on the synthesis of surfactin. Surfactin is a heptapeptide linked to a fatty acid via a cyclic lactone ring structure that is capable of inserting itself into cellular membranes where interacts with its lipid moiety leading to the loss of membrane barrier properties and eventually resulting in its complete disintegration by a detergent-

271 type mechanism (39). It has been widely reported that surfactin displays a significant antimicrobial activity $272(9,40)$. However, we found that the BsA01 post-ST variants show enhanced activity against the onion 273 pathogen S. terrestris but the biocontrol properties of the cell-free supernatant seemed to be independent 274 of surfactin in our study model. Most striking, B. subtilis appeared to have suppressed or reduced to a 275 minimum level the production of this classical antimicrobial lipopeptide after interacting with the fungus. 276 One could think that the lack of surfactin is a mere consequence of the metabolic change that arise along 277 with the phenotype variation experienced by BsA01 and that is not directly related to the antagonism per 278 se. However, it has been suggested that surfactins could interfere with the activity of other lipopeptides with 
279

280

281

282

283

284

285

286

287

288

289

290

291

292

293

294

295

296

proven antifungal activity such as fengycins/plipastatins and iturins $(38,41)$. In a study, $B$. amyloliquefaciens FZB42 mutants selectively repressed in the synthesis of one or two types of lipopeptides revealed that the co-production of surfactin and fengycin lowered the global antifungal activity (38). Such an effect of mixed lipopeptides was previously observed against the fungus Verticillium dahliae (42). Moreover, a Bacillus subtilis subsp. krictiensis surfactin-less mutant showed significantly higher antifungal activity against Fusarium oxysporum than its wild-type strain (41). Thus, we speculate that by reducing or preventing the biosynthesis of surfactin, BsA01 could avoid some negative impact on fitness while making an effective use of other inhibitory compounds against $S$. terrestris. However, we also found that of the main differences between pre and post-ST variants is a strong reduction in plipastatin abundance in the post-ST variants. Plipastatins are lipo-decapeptides with an internal lactone ring and with a $\beta$-hydroxy fatty acid chain (C14- C18) (43). Lipopeptides of this family are less hemolytic than surfactins but have a strong antifungal activity (43). Among the B. subtilis antibiome, cyclic lipopetides of surfactin and fengycin/plipastatin families are widely recognized as the main antimicrobials that can be secreted in biologically relevant amounts $(44,45)$. So, the striking reduction in the production of these two classical compounds in post-ST variants raises the question of what the essential antifungal factor that BsA01 produces to fight against $S$. terrestris is.

After repeated passages onto fresh growth media, the post-ST variants still maintained not only their colony morphology but also their increased ability to form biofilm and the absence of surfactin. This pointed out a possible participation of biofilm and/or surfactin-related pathways in the phenotype variation that characterize the BsA01 post-ST variants. Moreover, this observation indicates that $B$. subtilis ALBA01 undergoes a heritable phenotypic variation after interacting with the fungus in co-culture. Comparative whole genome as well as genomic pool-seq analysis between the BsA01 strain (pre-ST variant) and independently derived post-ST variants revealed mutations in the coding regions of the $\operatorname{com} A$ and $\operatorname{com} P$ genes, which are part of the quorum sensing system ComQXPA. In response to certain environmental stresses, the regulator ComA is activated by the kinase ComP, which is required for the transcription of several genes involved in competence development, antibiotic production, exopolysaccharide and degradative enzyme production as well as transport and fatty acid metabolism $(17,18)$. Among those genes, ComA activates the transcription of the srfA operon responsible for surfactin production $(15,46)$. 
307 Mutations found in comA gene in post-ST variants all consist of frameshift and non-sense mutations, which 308 result in the generation of premature stop codons and render truncated versions of the protein that losses 309 the predicted DNA-binding domain. It is then expected that this alteration produces the loss-of-function of 310 ComA and as a consequence the decrease or loss of surfactin-related genes expression. The same could 311 be speculated for the mutations found in the coding sequence of comP, which result in the loss of the 312 complete histidine kinase domain responsible of activating ComA. These observations highlight the 313 importance of the ComQXPA system in the evolutionary adaptation that BsA01 undergoes during the 314 interaction with $S$. terrestris in vitro. It has been described that Bacillus subtilis 916 strain was an effective biocontrol agent against the rice pathogen Rhizoctonia solani and that a mutant version of this strain, artificially obtained by means of lowenergy ion implantation, carries three missense mutations within the comA gene that the authors discuss to be the cause of the lower levels of surfactin produced by the mutant. However, in this work in opposition to what we observe, the reduction in surfactin production leads to a non-optimal performance of $B$. subtilis 916 against the fungus (47). Bacillus subtilis has an average of $4-5 \%$ of its genome devoted to antibiotic 321 synthesis and has the potential to produce more than two dozens of structurally diverse antimicrobial compounds. More than $10 \%$ of its genome is controlled by the sophisticated ComQXPA quorum sensing system centered on the transcriptional regulator, ComA. It has also been found that numerous genes with unknown function were also affected by ComA (18). As mentioned before, the abrogation of surfactin in post-ST variants might be explained by a loss-of-function of mutations in the comA gene. But, how could mutations in comQXPA genes explain the reduction in the production of plipastatin also observed in the 327 post-ST variants? ComQXPA system has also been described to be involved in the transcription of the 328 pleiotropic regulator DegQ, which controls the production of degradative enzymes and is necessary for 329 plipastatin production $(21,48,49)$. Thus, we hypothesize that the reduction in plipastatin might represent 330 an indirect effect of the mutations in $\operatorname{com} A$ and $\operatorname{comP}$ genes through alteration in $\operatorname{deg} Q$ expression. 331 Interestingly, co-cultures have also been used to explore the modulation of quorum sensing in the search 332 for new antibiotics since quorum sensing molecules play effective regulatory roles within bacterial 333 populations by adapting global growth to the level of nutrient availability $(50,51)$. However, at present no 334 quorum sensing response mutants were observed in natural populations of Bacillus subtilis. Most bacterial 
species are confronted with frequent and often extreme changes in environmental conditions to which they must rapidly adapt. To this end, they operate diverse regulatory networks being one of the most important

337 involved in quorum sensing control, which in response to particular environmental signals brings global

338 changes in gene expression that in turn regulate complex physiological behaviors and confer adaptability and competiveness (52). As of yet, reports have demonstrated neither a mutational evolution of the ComA

340 protein mediating a stable phenotypic change nor the role of ComQXPA mutations in positive expression 341 or proper activity/functioning of extracellular antifungal metabolites that could mediate an adaptive response 342 in B. subtilis.

Taken together, our findings allows us to speculate that the mutation found in the ComQXPA system of $B$. subtilis post-ST variants may be involved in a regulatory mechanism that leads to the activation of a cryptic

345 biosynthetic pathway, which specifically responds to extracellular signals involved in the interaction

346 between the $B$. subtilis strain and $S$. terrestris in co-cultures. Future investigations will be necessary to 347 expand our knowledge on the identity and the role that the antagonistic metabolites play in this iteraction348 driven evolution process.

\section{Materials and Methods}

350 Materials and methods are described in the SI Appendix, including microorganisms growth conditions, $B$. 351 subtilis mutants construction, phenotyping characterization analysis, whole genome sequencing and pool352 seq analysis and metabolomics studies based on liquid chromatography - tandem mass spectrometry and 353 nuclear magnetic resonance spectrometry.

\section{Acknowledgments}

355 The authors are grateful to Dr. Alejandro Moyano for valuable discussion and comments on the manuscript.

356 We would like to thank Consejo Nacional de Investigaciones Científicas y Técnicas (CONICET), Agencia 357 Nacional de Promoción Científica y Tecnológica (ANPCyT). This work was supported by by MINCyT | 358 Agencia Nacional de Promoción Científica y Tecnológica (ANPCyT), grant PICT 2013-2592 to A.G.A.O and 359 PICT 2012-2088 to A.M.S.; the US National Science Foundation (NSF) Inspire Track II IOS-1343020; 
National Institutes of Health (NIH) Grants GMS10RR029121, 5P41GM103484-07 and the Deutsche

361 Forschungsgemeinscht (DFG) with Grant PE 2600/1.

\section{Author Contributions}

A.G.A.O, A.M.S, D.P., D.A.D and P.C.D. designed the experiments and supervised the study. A.G.A.O, performed DNA isolation and analyzed bioinformatics data of bacterial genomes. A.G.A.O. analyzed NMR data. A.G.A.O. and D.P. collected the mass spectrometry data. A.G.A.O., D.P. and P.C.D. analyzed the

367 metabolomics data and performed molecular networking. A.G.A.O. and A.M.S. integrated all experimental data and performed the final interpretation. A.G.A.O., A.M.S., D.P. and D.A.D. wrote the paper. All authors

369 discussed, edited and agreed on the final manuscript.

\section{References}

371 1. Kolter R \& Greenberg EP (2006) Microbial sciences: the superficial life of microbes. Nature 441(7091):300-302.

3732 2. Schroeckh V, et al. (2009) Intimate bacterial-fungal interaction triggers biosynthesis of archetypal polyketides in Aspergillus nidulans. Proceedings of the National Academy of Sciences 106(34):14558-14563.

376 3. Onaka H, Mori Y, Igarashi Y, \& Furumai T (2011) Mycolic acid-containing bacteria induce natural377 product biosynthesis in Streptomyces species. Applied and environmental microbiology 77(2):400$378 \quad 406$.

379 4. Straight PD \& Kolter R (2009) Interspecies Chemical Communication in Bacterial Development. $380 \quad$ Annual Review of Microbiology 63(1):99-118.

381 5. Ryan RP \& Dow JM (2008) Diffusible signals and interspecies communication in bacteria. $382 \quad$ Microbiology 154(7):1845-1858.

383 6. López D, Vlamakis H, Losick R, \& Kolter R (2009) Paracrine signaling in a bacterium. Genes \& Development 23(14):1631-1638. 
385 7. Branda SS, Chu F, Kearns DB, Losick R, \& Kolter R (2006) A major protein component of the Bacillus subtilis biofilm matrix. Molecular Microbiology 59(4):1229-1238.

387

8. Vlamakis H, Aguilar C, Losick R, \& Kolter R (2008) Control of cell fate by the formation of an architecturally complex bacterial community. Genes \& Development 22(7):945-953.

389

9. Bais HP, Fall R, \& Vivanco JM (2004) Biocontrol of Bacillus subtilis against Infection of Arabidopsis Roots by Pseudomonas syringae Is Facilitated by Biofilm Formation and Surfactin Production. Plant

10. Ongena M \& Jacques P (2008) Bacillus lipopeptides: versatile weapons for plant disease biocontrol. Trends in Microbiology 16(3):115-125.

11. B. KD \& Richard L (2003) Swarming motility in undomesticated Bacillus subtilis. Molecular Microbiology 49(3):581-590.

12. Kinsinger RF, Shirk MC, \& Fall R (2003) Rapid surface motility in Bacillus subtilis is dependent on extracellular surfactin and potassium ion. Journal of Bacteriology 185(18):5627-5631.

13. Julkowska D, Obuchowski M, Holland IB, \& Séror SJ (2005) Comparative analysis of the development of swarming communities of Bacillus subtilis 168 and a natural wild type: critical effects of surfactin and the composition of the medium. Journal of Bacteriology 187(1):65-76.

15. Roggiani M \& Dubnau D (1993) ComA, a phosphorylated response regulator protein of Bacillus subtilis, binds to the promoter region of srfA. Journal of Bacteriology 175(10):3182-3187.

16. Nakano MM \& Zuber P (1991) The primary role of comA in establishment of the competent state in Bacillus subtilis is to activate expression of srfA. Journal of Bacteriology 173(22):7269-7274. 
413 19. Hamoen LW, Eshuis H, Jongbloed J, Venema G, \& Sinderen D (1995) A small gene, designated coms, located within the coding region of the fourth amino acid-activation domain of $\operatorname{srf} A$, is

20. Ogura M, et al. (2002) Whole-genome analysis of genes regulated by the Bacillus subtilis competence transcription factor ComK. Journal of Bacteriology 184(9):2344-2351.

21. Tsuge K, Ano T, Hirai M, Nakamura Y, \& Shoda M (1999) The Genes degQ, pps, and Ipa-8 (sfp) are responsible for conversion of Bacillus subtilis 168 to plipastatin production. Antimicrobial Agents and Chemotherapy 43(9):2183-2192.

22. Tsuge K, Matsui K, \& Itaya M (2007) Production of the non-ribosomal peptide plipastatin in Bacillus

23. Vanittanakom N, Loeffler W, Koch U, \& Jung G (1986) Fengycin-a novel antifungal lipopeptide

24. Falardeau J, Wise C, Novitsky L, \& Avis T (2013) Ecological and mechanistic insights into the direct

25. Cawoy $\mathrm{H}$, et al. (2015) Lipopeptides as main ingredients for inhibition of fungal phytopathogens by Bacillus subtilis/amyloliquefaciens. Microbial Biotechnology 8(2):281-295.

26. Tang Q, et al. (2014) Effects of fengycin from Bacillus subtilis fmbJ on apoptosis and necrosis in Rhizopus stolonifer. Journal of Microbiology 52(8):675-680.

433 27. Shank EA, et al. (2011) Interspecies interactions that result in Bacillus subtilis forming biofilms are that cause potassium leakage trigger multicellularity in Bacillus subtilis. Proceedings of the National Academy of Sciences of the United States of America 106(1):280-285. 
29. Zeriouh H, de Vicente A, Pérez-García A, \& Romero D (2014) Surfactin triggers biofilm formation of Bacillus subtilis in melon phylloplane and contributes to the biocontrol activity. Environmental Microbiology 16(7):2196-2211.

30. Albarracín Orio AG, Brücher E, \& Ducasse DA (2016) A strain of Bacillus subtilis subsp. subtilis shows a specific antagonistic activity against the soil-borne pathogen of onion Setophoma terrestris. European Journal of Plant Pathology 144(1):217-223.

31. Shemesh M \& Chai Y (2013) A Combination of Glycerol and Manganese Promotes Biofilm Formation in Bacillus subtilis via Histidine Kinase KinD Signaling. Journal of Bacteriology 195(12):2747-2754

32. Watrous J, et al. (2012) Mass spectral molecular networking of living microbial colonies. Proceedings of the National Academy of Sciences 109(26):E1743-E1752.

33. Wang M, et al. (2016) Sharing and community curation of mass spectrometry data with Global Natural Products Social Molecular Networking. Nature Biotechnology 34:828.

34. Nakano MM, et al. (1991) srfA is an operon required for surfactin production, competence development, and efficient sporulation in Bacillus subtilis. Journal of Bacteriology 173(5):17701778.

35. Kearns DB, Chu F, Branda SS, Kolter R, \& Losick R (2005) A master regulator for biofilm formation by Bacillus subtilis. Molecular Microbiology 55(3):739-749.

36. Branda SS, González-Pastor JE, Ben-Yehuda S, Losick R, \& Kolter R (2001) Fruiting body formation by Bacillus subtilis. Proceedings of the National Academy of Sciences 98(20):1162111626.

37. Finn RD, et al. (2017) InterPro in 2017-beyond protein family and domain annotations. Nucleic Acids Research 45(D1):D190-D199.

38. Zihalirwa Kulimushi P, Argüelles Arias A, Franzil L, Steels S, \& Ongena M (2017) Stimulation of Fengycin-Type Antifungal Lipopeptides in Bacillus amyloliquefaciens in the Presence of the Maize Fungal Pathogen Rhizomucor variabilis. Frontiers in Microbiology 8(850). 
465 39. Carrillo C, Teruel JA, Aranda FJ, \& Ortiz A (2003) Molecular mechanism of membrane permeabilization by the peptide antibiotic surfactin. Biochimica et Biophysica Acta (BBA) Biomembranes 1611(1):91-97.

40. Fan $\mathrm{H}$, et al. (2017) Biocontrol of Bacterial Fruit Blotch by Bacillus subtilis 9407 via SurfactinMediated Antibacterial Activity and Colonization. Frontiers in Microbiology 8(1973).

470 41. Kim YT, et al. (2017) Organization and characterization of genetic regions in Bacillus subtilis subsp. krictiensis ATCC55079 associated with the biosynthesis of iturin and surfactin compounds. PLoS ONE 12(12):e0188179.

42. Liu J, Hagberg I, Novitsky L, Hadj-Moussa H, \& Avis TJ (2014) Interaction of antimicrobial cyclic

43. Steller S, et al. (1999) Structural and functional organization of the fengycin synthetase

44. Kinsella K, P. Schulthess C, Morris T, \& D. Stuart J (2009) Rapid quantification of Bacillus subtilis antibiotics in the rhizosphere pp 374-379.

45. Debois D, et al. (2014) Spatiotemporal monitoring of the antibiome secreted by Bacillus biofilms on plant roots using MALDI mass spectrometry imaging. Analytical chemistry 86(9):4431-4438.

46. Nakano MM, Xia LA, \& Zuber P (1991) Transcription initiation region of the srfA operon, which is controlled by the comP-comA signal transduction system in Bacillus subtilis. Journal of Bacteriology 173(17):5487-5493.

47. Wang X, et al. (2010) Three non-aspartate amino acid mutations in the ComA Response regulator

48. Msadek T, Kunst F, Klier A, \& Rapoport G (1991) DegS-DegU and ComP-ComA modulator-effector pairs control expression of the Bacillus subtilis pleiotropic regulatory gene degQ. Journal of Bacteriology 173(7):2366-2377.

49. Wang $P$, et al. (2015) DegQ regulates the production of fengycins and biofilm formation of the biocontrol agent Bacillus subtilis NCD-2. Microbiological Research 178:42-50. 
493 50. Bertrand S, et al. (2014) Metabolite induction via microorganism co-culture: A potential way to 494 enhance chemical diversity for drug discovery. Biotechnology Advances 32(6):1180-1204.

495 51. Charusanti P, et al. (2012) Exploiting Adaptive Laboratory Evolution of Streptomyces clavuligerus $496 \quad$ for Antibiotic Discovery and Overproduction. PLOS ONE 7(3):e33727.

497 52. Miller MB \& Bassler BL (2001) Quorum sensing in bacteria. Annual Reviews in Microbiology $498 \quad 55(1): 165-199$. 
A

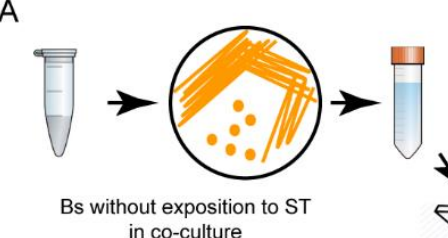

in co-culture

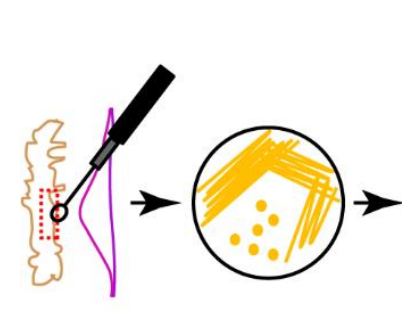

Recovery of Bs after co-culture wth ST

C
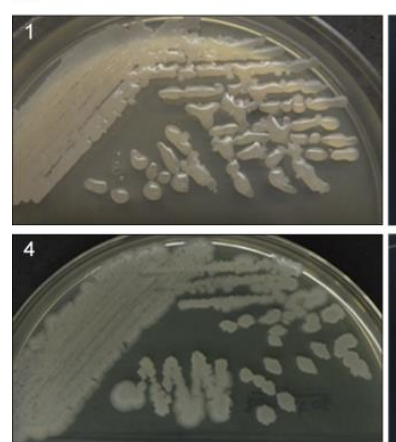

Obtention of cell-free supernatants
B

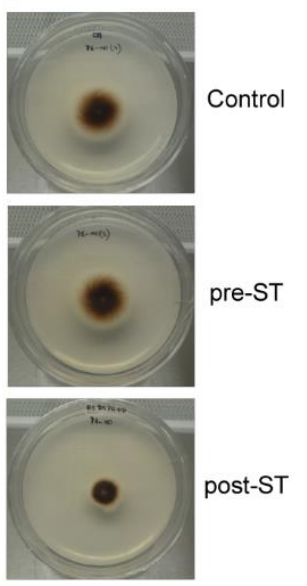

ost-ST

pre-ST

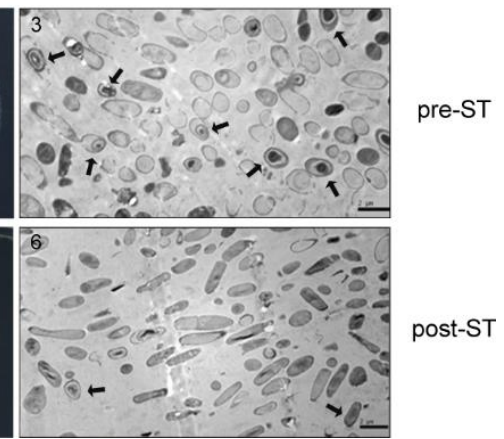

501

502

503

504

505

506

507

508

509

510

511

512

513

514

515

516

517

518

Figure 1. A specific induced phenotype change was observed in B. subtilis upon the interaction with S. terrestris.

After 15 days of co-culture with $S$. terrestris (ST) in vitro, a sample of the B. subtilis A01 (BsA01) colony was taken, stored and named post-ST variant to differentiate it from its original version never exposed to ST in co-culture (pre-ST variant). (A) The antifungal activity of cell-free supernatants (SN) of both variants were assayed according to the workflow shown. (B) A high growth inhibition of $S$. terrestris was observed in dishes containing only SN of post-ST. The figure shows the fungal growth inhibition at 4 days after inoculation. (C)The interaction with the fungus also leads BsA01 to experience a phenotypic change since post-ST variants develop into a rougher and wrinkled colonies in LB agar compared to the pre-ST variant. Panels 2 and 5 show pictures of individual colonies of a pre-ST and a post-ST variant, respectively, and panels 1 and 4 their respective agar plates showing the overall macroscopic appearance of each variant culture. Electron microscopy of $B$. subtilis post-ST variants exposed an increment in the number of longer cells and a reduction of the number of sporulated cells (panel 6) in contrast to control cells never exposed to the fungus or pre-ST variants (panel 3) (bar scale, $2 \mu \mathrm{m}$ ). Arrows in panels 3 and 6 indicate sporulated cells. 
A
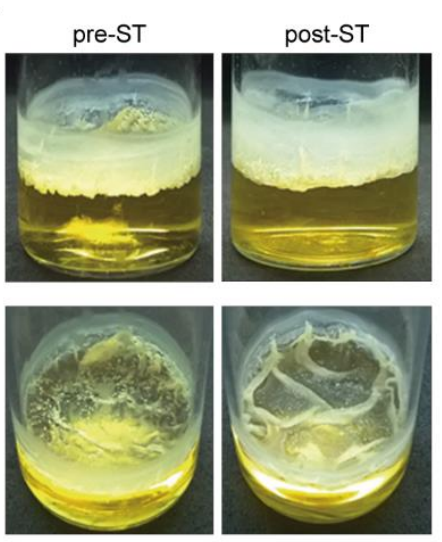

C

pre-ST

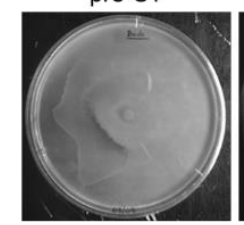

post-ST

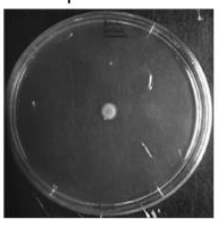

B
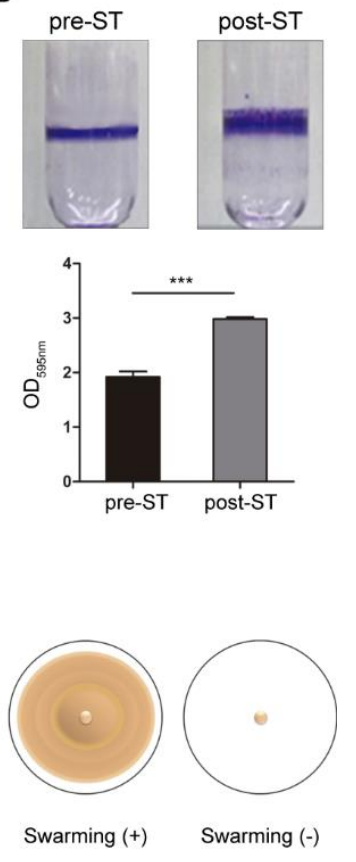

\section{Figure 2. B. subtilis post-ST variants form robust biofilm and lacks swarming motility.}

(A) B. subtilis post-ST variants were able to develop into pellicles characteristically associated with strong biofilm formation in liquid medium while, surprisingly, the pre-ST variant formed pellicles with diminished wrinkles. (B) The quantification of biofilm formation in borosilicate surfaces by using crystal violet staining also revealed an increase in biofilm formation in B. subtilis post-ST variants compared to the pre-ST variant. (C) Swarming motility was assessed in $0.7 \%$ agar LB plates. The $B$. subtilis pre-ST variant occupied the entire plate showing the expected full motility while $B$. subtilis post-ST variants clearly showed a swarming deficiency as the colony could not develop and extend beyond the region of inoculation. 
B

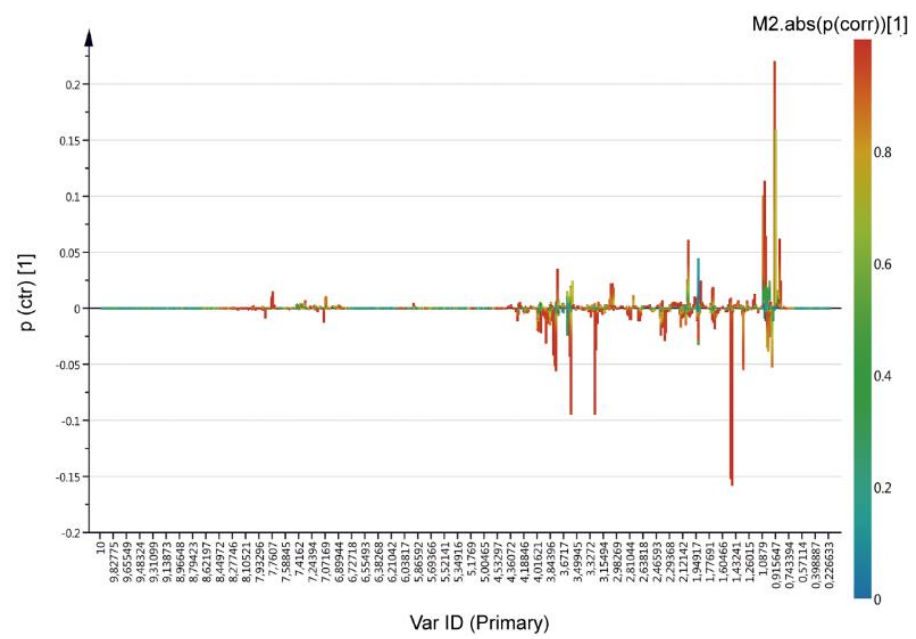

Var ID (Primary)

Figure 3. A metabolomics profile variation in B. subtilis ALBA01 is observed after the interaction with S. terrestris in vitro.

A

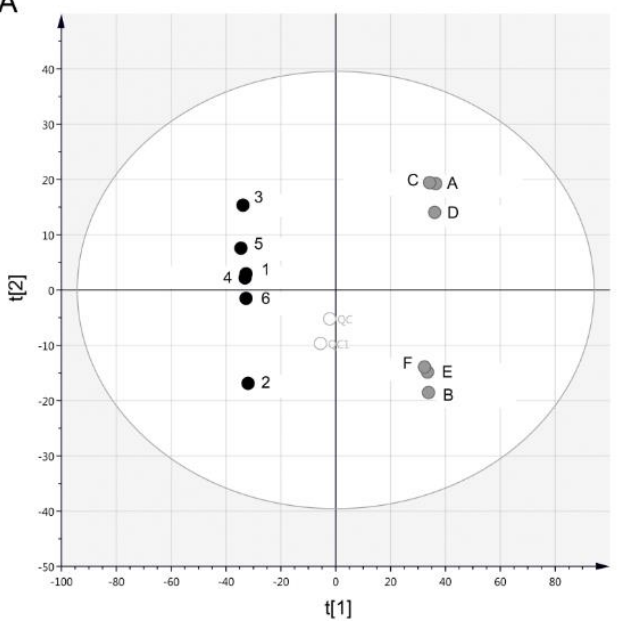

The high antifungal activity found in cell-free supernatants (SN) of post-ST variants appeared to be associated to changes in the metabolomics profile of released compounds since differences in SN between the pre and post-ST variants could be observed by an untargeted NMR approach. (A) The PCA scores plot shows a clear separation of the SN samples according to their type, pre-ST variant (black) and post-ST variant (grey) samples, in the first two principal components derived from the $1 \mathrm{H}$ NMR spectra of Bacillus conditioned media (percent variation in the NMR data explained by the model, $R 2 x=70.4 \%$; percent variation in the NMR data predicted by the model from cross validation, $Q 2 x=57 \%$, based on a 2 components model). Quality control (QC) samples are shown as white circles. (B) OPLS-DA S-line plots with pairwise comparison of data from NMR spectra obtained from Bacillus variants SN. Colors are associated with the correlation of metabolites characterized from the $1 \mathrm{H}$ NMR data for the class of interest with the scale shown on the right-hand side of the plot. 
A

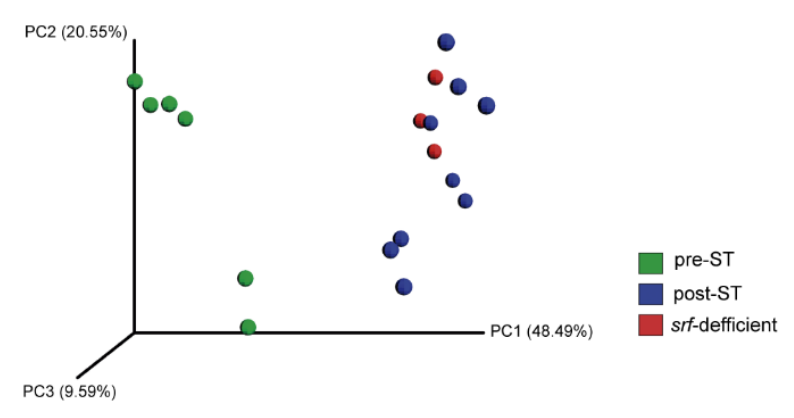

C

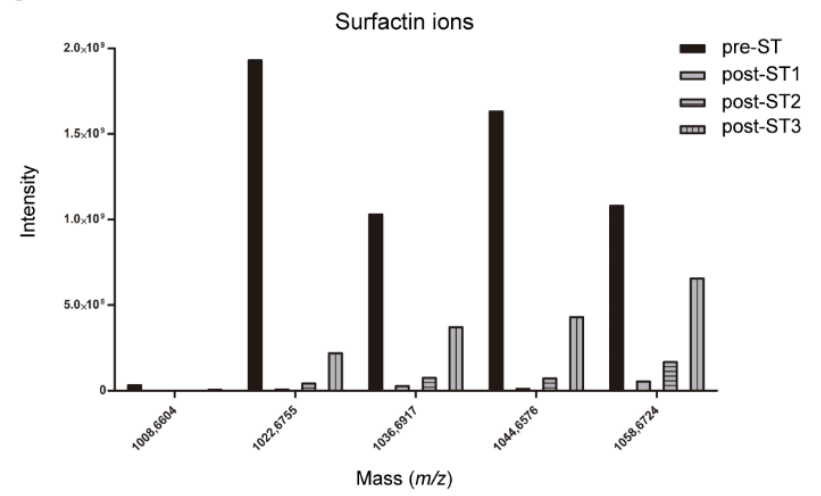

B

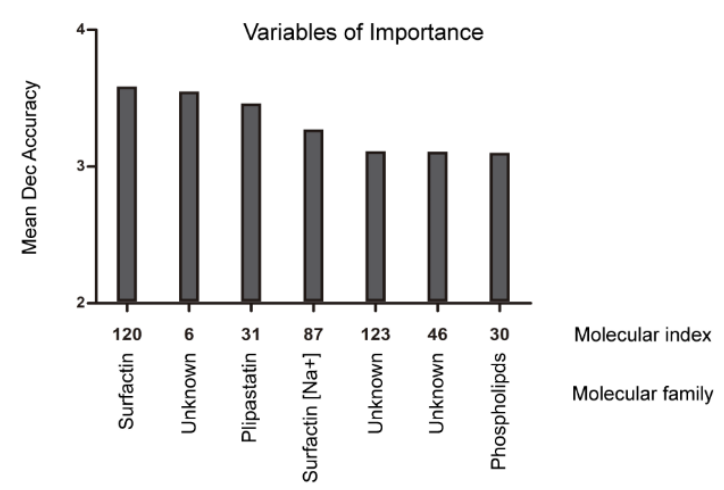

D

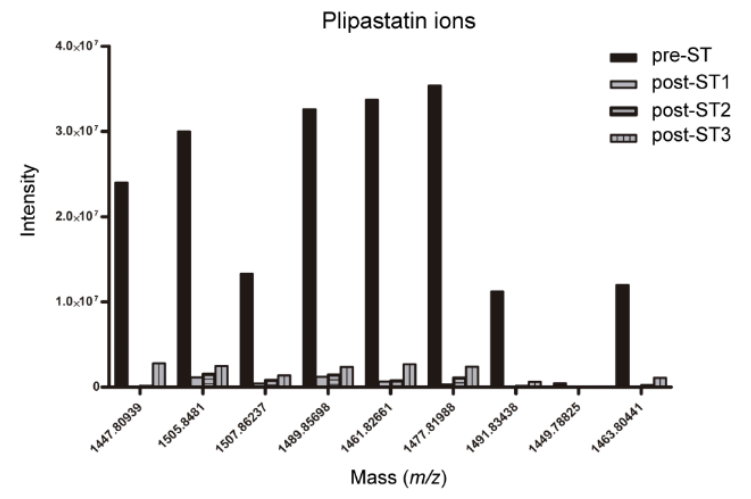

Figure 4. LC-MS/MS based metabolomics exposed the chemical signatures that differentiate the $B$. subtilis pre and post-ST variants.

555 (A) A strong separation between the pre and post-ST variants could be observed in PcoA plots. (B) Random Forest analysis revealed that surfactin and plipastatin were one of the most important variables that determined this separation. The post-ST variants showed a marked reduction in the intensity of surfactin (C) and plipastatin ions (D).

559 
A

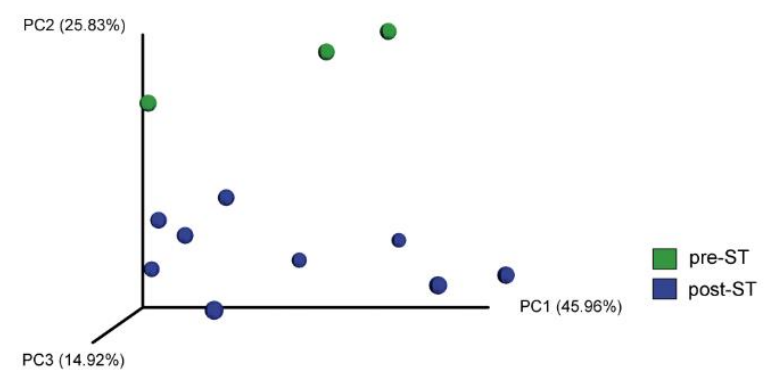

C

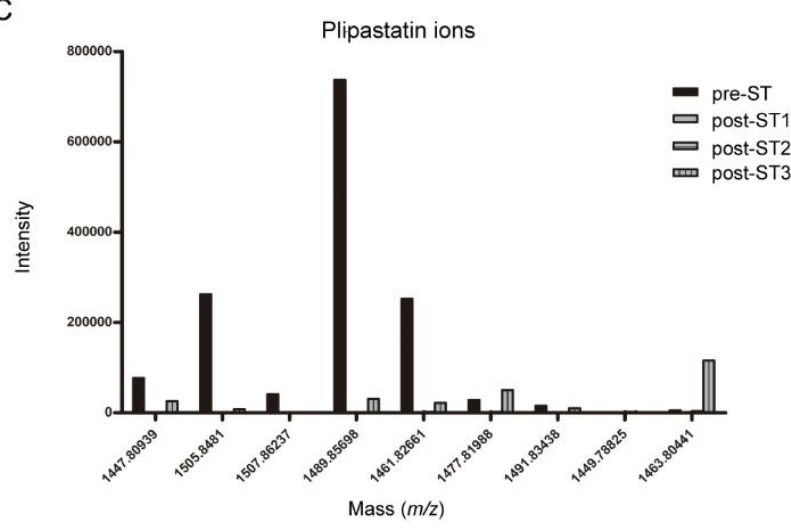

B

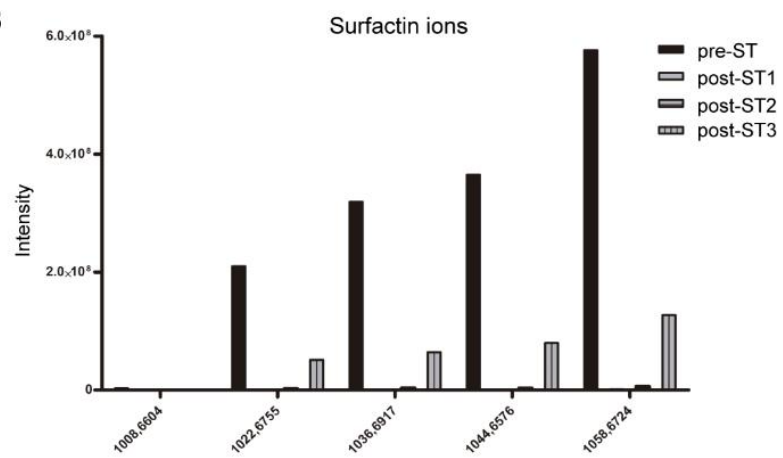

D

Mass $(m / z)$

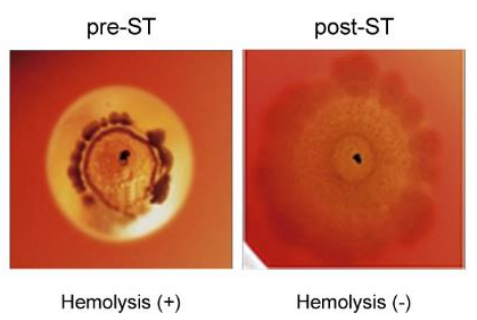

Figure 5. LC-MS/MS metabolomics analysis of the cell-free supernatants of $B$. subtilis pre and postST variants.

(A) PcoA plots show a clear discrimination according to the origin of the cell-free supernatants, with postST samples clustering separated from pre-ST variants. Surfactin (B) and plipastatin (C) could not be detected in post-ST cell-free supernatants in levels comparable to those found in the pre-ST variants. (D) Consistent with the fact that apparently the post-ST variants have lost the ability to produce surfactin, they did not show the typical hemolytic activity associated to this lipopeptide. 


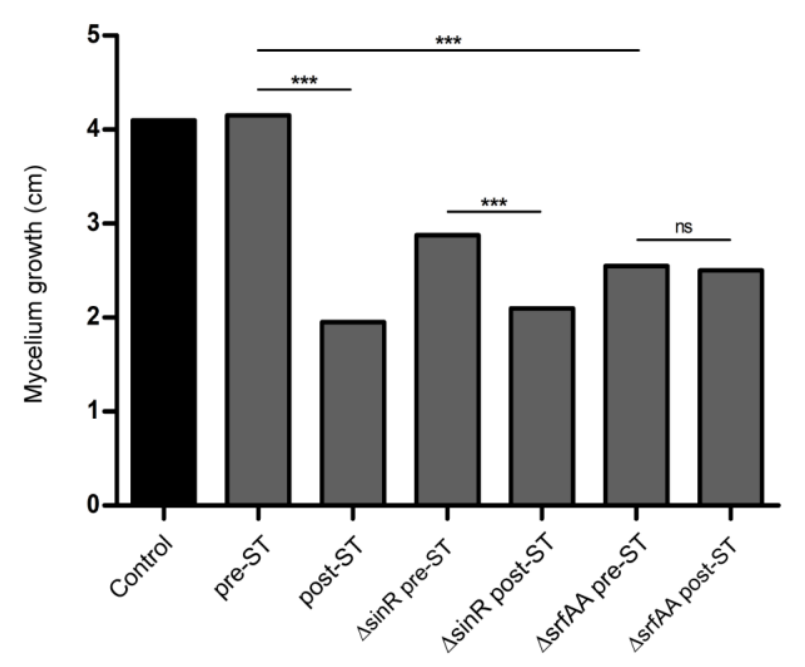

Figure 6. Biofilm correlates with the antagonism and surfactin is not the metabolite responsible of the induced antifungal activity.

The activity of cell-free supernatants of BsA01 $\Delta \sin R$ and $\triangle s r f A$ mutants, pre- and post-contact with $S$. terrestris, was screened in co-culture as described in the text. Graph columns represent mean values of mycelium growth. The experiments were independently repeated at least four times. ${ }^{*}$ indicates that the mycelium growth is significantly different between the strains tested and ns denotes no significant differences (Tukey's Multiple Comparison Test, $p$-value $<0.0001$ ). The antifungal activity of the cell-free supernatants of the surfactin deficient $\triangle$ srfAA mutant pre- and post-contact with the fungus were similar but lower than those exhibited by the BsA01 post-ST variants and the $\Delta \sin R$ mutant indicating that the inability to produce surfactin cannot completely abrogate the antifungal activity of $B$. subtilis ALBA01. 


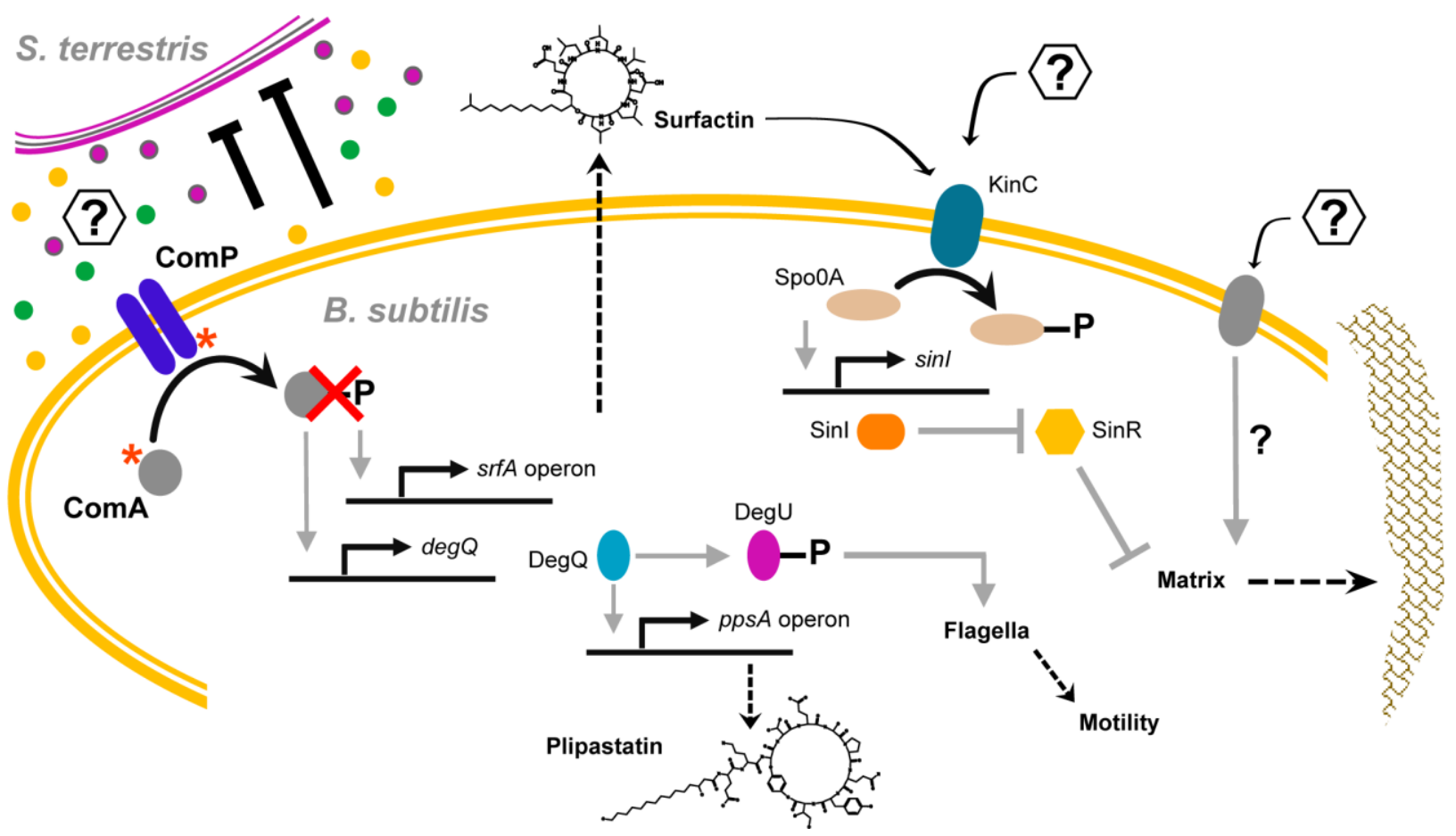

Figure 7. Schematic representation of the interaction between $S$. terrestris and B. subtilis and its influence on the cellular processes implied in the mutation-based phenotypic variation experienced by the bacterium. The phenotypic variation observed in $B$. subtilis after interacting with the fungus manifests an enhanced antifungal activity in cell-free supernatants, biofilm formation and deficient swarming motility. Metabolic exchange and the chemical scenario defined during the confrontation of the two individuals (fungus and bacterium) drive a mutational event and selection of $B$. subtilis cells that carry truncated versions of either the master regulator ComA and/or the kinase ComP (represented by asterisks). The absence of the activated forms of this two key protein components of the ComQXPA quorum-sensing system results in a lack of activation of the expression of ComA-controlled genes among which are the surfactin-encoded operon and $\operatorname{deg} Q$. Transcription of $\operatorname{deg} Q$ is necessary for the production of the nonribosomal lipopeptide, plipastatin. Besides, DegQ positively regulates motility-associated genes through modulation of the activity of the pleiotropic factor DegU. Surfactin has been described as a signaling molecule that induces the activation of the Sinl-SinR pathway causing extracellular matrix production and biofilm formation. The effect of its abrogation might be overcome by the action of some particular chemical signal, produced during the interaction, which is able to either activate the phosphorilation of Spo0A, and thus matrix gene expression, or act via a still unknown mechanism that finally results in the biofilm formation characteristically observed in $B$. subtilis after interacting with $S$. terrestris. The physicochemical conditions that arose during the interaction may contribute to the adaptation of $B$. subtilis and the improvement of its antagonistic behavior towards $S$. terrestris. 


\section{Tables}

614 Table 1. Whole genome sequencing comparative analysis of $B$. subtilis post-ST variants revealed mutations

615 in comQXPA coding regions

616

\begin{tabular}{|c|c|c|c|c|}
\hline Variant & Scaffold/position & Gene & $\begin{array}{l}\text { Nucleotide } \\
\text { substitution }\end{array}$ & Description \\
\hline Post-ST 1 & $1 / 69978$ & $\operatorname{com} A$ & Ins A 415 & $\begin{array}{l}\text { Transcriptional regulatory protein } \\
\text { ComA }\end{array}$ \\
\hline Post-ST 2 & $1 / 69978$ & $\operatorname{com} A$ & Ins A 415 & $\begin{array}{l}\text { Transcriptional regulatory protein } \\
\text { ComA }\end{array}$ \\
\hline Post-ST 2 & 2/165291 & $y h f V$ & $\Delta \mathrm{A} 1089$ & $\begin{array}{l}\text { Methyl-accepting chemotaxis } \\
\text { protein }\end{array}$ \\
\hline Post-ST 2 & $3 / 371952$ & spoOA & G289C & $\begin{array}{l}\text { Sporulation two-component } \\
\text { response regulator }\end{array}$ \\
\hline Post-ST 3 & $1 / 71309$ & comP & $\Delta 632-731$ & $\begin{array}{l}\text { Two-component sensor kinase } \\
\text { ComP }\end{array}$ \\
\hline
\end{tabular}

618 Table 2. Mutations found in $\operatorname{comPQXA}$ operon by whole-genome sequencing of pools of individuals

619 experiment (Pool-seq)

620

621

\begin{tabular}{llll}
\hline $\begin{array}{l}\text { Scaffold } \\
\text { (ALBA01) }\end{array}$ & Position & Gene & $\begin{array}{l}\text { Nucleotide } \\
\text { Substitution }\end{array}$ \\
\hline 1 & 69979 & comA & C601T \\
1 & 69978 & comA & Ins A 415 \\
1 & 70101 & $c o m A$ & Ins CTGGG 299 \\
1 & 70185 & $c o m A$ & T215A \\
1 & 71264 & $c o m P$ & Ins CG 1517 \\
1 & 71919 & $c o m P$ & Ins AT 863 \\
\hline
\end{tabular}

